\title{
UNIVERSITARIOS ACTORES EN LA PRÁCTICA DE VALORES
}

\section{UNIVERSITY ACTORS IN THE PRACTICE OF VALUES}

Tania Yuliza López Jiménez*, Gladys Hernández Romero**

\begin{abstract}
*Estudiante de la Licenciatura en Administración. Universidad Juárez Autónoma de Tabasco. División Académica de Ciencias Económico Administrativas. ORCID: https://orcid.org/0000-0003-3117-3097

**Doctora en Educación. Profesora Investigadora. Universidad Juárez Autónoma de Tabasco. División Académica de Ciencias Económico Administrativas. Email: gladiolita6@hotmail.com ORCID: https://orcid.org/0000-0002-9045-2937
\end{abstract}

Dirección para recibir correspondencia: tanialopz2015@gmail.com

Fecha de recibido: 8 de noviembre de 2018

Fecha de aceptación: 20 de noviembre de 2018 


\section{UNIVERSITARIOS ACTORES EN LA PRÁCTICA DE VALORES}

\section{RESUMEN}

OBJETIVO: Exponer los resultados de un estudio sobre la práctica de los valores en universitarios de primero y segundo ciclo de la División Académica de Ciencias Económico Administrativas (DACEA) de la Universidad Juárez Autónoma de Tabasco (UJAT) en México.

MATERIAL Y MÉTODO: El instrumento empleado para la obtención de datos fue un cuestionario estructurado, tomando una muestra para su aplicación de 261 estudiantes, la cual se determinó de forma intencionada mediante el método cualitativo, el cual consiste en proveer datos descriptivos de aquellos aspectos impalpables del comportamiento del ser humano y de la vida, como las creencias y actitudes.

RESULTADOS: Se muestran que más de la mitad de los alumnos siempre practican los valores hacia sus padres y profesores, pero los practican menos cuando se trata de sus compañeros.

CONCLUSIONES: Existe la necesidad de concientizarlos respecto a la importancia que tienen los valores en su vida personal y profesional.

PALABRAS CLAVE: Educación superior. Valores. Formación. Transformación social.

\section{ABSTRACT}

OBJECTIVE: To present the results of a study on the practice of values in university students of the first and second cycle of the Academic Division of Economic and Administrative Sciences (DACEA) from the Universidad Juárez Autónoma de Tabasco (UJAT) in Mexico.

MATERIAL AND METHOD: The instrument used to obtain data was a structured questionnaire, taking a sample for its application of 261 students, which was determined intentionally by the qualitative method that consists on providing descriptive data of those impalpable aspects of the behavior of the human being and the life, such as beliefs and attitudes.

RESULTS: It is shown that more than half of the students always practice the values towards their parents and teachers, but they practice these values less towards their classmates.

CONCLUSIONS: There is a need to raise awareness in the students about the importance of values in their personal and professional lives.

KEY WORDS: Higher education. Values. Training. Social transformation. 


\section{UNIVERSITARIOS ACTORES EN LA PRÁCTICA DE VALORES}

\section{INTRODUCCIÓN}

Hoy en día, es importante reforzar en universitarios los valores que han adquirido con anterioridad y los nuevos que están por desarrollar, de esta manera se busca moldear personas con participación crítica y comprometida con la transformación social. Mejía (2007) señala que el desafío que requiere la sociedad va más allá de brindar conocimiento y habilidades, se debe buscar formar profesionales como personas íntegras.

De igual forma, Aranda y Salgado (2005) mencionan que las Instituciones de Educación Superior (IES), al contar con una misión, deben crear un contexto adecuado para propiciar la adopción de conductas que pongan de manifiesto una formación sólida en los valores humanos.

Toda educación basada en valores debe permitir a los alumnos tener una actitud reflexiva, de juicio y de consciencia en su tarea, permitiéndoles tener claro su futuro y disponerse a enfrentar los retos que demanda toda sociedad (Montesinos, 2010).

“Educar en valores, es la acción planificada, intencionada, orientada y controlada que ejercen la familia, la escuela y la comunidad mediante el ejemplo personal en las actuaciones de los adultos responsabilizados con el encargo social de la formación de las nuevas generaciones" (Báxter, 2009).

Para concretar esta información, se revisaron autores como Mazadiego (2005) que señala los valores como el resultado de las experiencias individuales que vive el ser humano y que interpreta de acuerdo a una autovaloración. González y Cardentey (2016) exponen la existencia de condiciones que debe seguir el docente para asegurar una enseñanza de calidad, entre otros autores de gran aporte al artículo.

Como parte de la investigación, se aplicaron 261 cuestionarios en las instalaciones de la Ciudad del Conocimiento perteneciente a la División Académica de Ciencias Económico Administrativas (DACEA), de la Universidad Juárez Autónoma de Tabasco (UJAT), con la finalidad de revelar si los alumnos practican los valores hacia sus padres, sus profesores, compañeros, así como conocer donde se forjan los valores, cual es el valor de más importancia para ellos y si sus profesores le hablan acerca de los valores. 


\section{UNIVERSITARIOS ACTORES EN LA PRÁCTICA DE VALORES}

\section{Revisión literaria}

Mazadiego (2005) afirma que los valores son producto de las experiencias y que una vez adquiridos por el individuo, estos mismos, le sirven de guía en su conducta. Como todos sabemos la base fundamental en los que se inserta a la persona la noción de valorar, es en casa, guiados por los padres.

No obstante, cuando este individuo llega a la etapa de formación profesional requiere de una guía para adquirir los conocimientos, habilidades y desarrollar competencias, que por ende debe reforzar con valores éticos que le permitan desempeñarse en un área de trabajo.

Tam (2002), Mahony (2009) y Benito (2006) en De los Ríos, Millán, Ruíz y Tirado (2015) señalan que la misión de las instituciones de educación superior va más allá del crecimiento intelectual, exhibiendo que también debe fortalecer el desarrollo social, emocional y cultural.

Por ello, se busca crear mediante la enseña una cultura basada en valores, que permita avanzar en el desarrollo de matrices singulares y personales guiados por ideales como la dignidad, la libertad y la justicia de forma que hagan posibles realidades sociales inclusivas (Martínez, 2006).

Por otro lado, la ética permite tener argumentos fundamentados acerca del comportamiento moral de los seres humanos, tomado de la mano de los valores que practican (Bolívar, 2005). En este sentido, las universidades tienen el compromiso de moldear al estudiante, para que éste sea un buen profesionista y refleje en un puesto de trabajo y en la sociedad, el conocimiento, las competencias y los valores adquiridos.

González y Cardentey (2016) señalan que existen condiciones necesarias para la educación de valores, tres de ellas son:

1. Caracterizar al estudiante en cuanto a determinantes internas de la personalidad como intereses, valores, actitudes y proyecto de vida.

2. Conocer los entornos familiar, universitario y social donde se desempeñan los estudiantes para poder determinar sus actuaciones y necesidades formativas de valores.

3. Definir modelos de educación de valores para los estudiantes universitarios. 


\section{UNIVERSITARIOS ACTORES EN LA PRÁCTICA DE VALORES}

Obtener buenos resultados en la aplicación de estas condiciones, no sólo dependerá del profesorado sino también del estudiante, quien debe aceptarlas para su formación profesional y para esto, el educando necesita desarrollar la capacidad valorativa, que conlleve a generar resultados positivos hacia la sociedad.

En un estudio realizado por la Dra. Viviana González Maura, con el objetivo de elaborar un proyecto de trabajo educativo para la formación de valores en los estudiantes, se demostró que el docente universitario es un especialista en su profesión, pero carece de formación psicopedagógica (González, 2000).

En este sentido, no se debe improvisar la formación integral de los alumnos, lo que de hecho exige pensar en la formación del profesorado universitario, su criterio, su testimonio, su idoneidad y la capacidad de involucrar y comprometer a los estudiantes en un proyecto ético con repercusiones en la vida personal y socio-cultural (Bilbeny, 1997) en Mejía (2007).

Riera y Sansevero (2013) consideran que el estudiante debe tener el compromiso de actuar con respeto, honestidad y responsabilidad durante las orientaciones impartidas por el profesor, las cuales faciliten una experiencia de aprendizaje que promueva el interés de seguir actuando positivamente en correspondencia a las necesidades de su comunidad.

Los universitarios tienen el papel protagónico de su propia formación profesional; es decir, que no todo el trabajo recae en como sea la educación que brinde la universidad o la enseñanza del profesor hacia el alumno, sino que está en ellos el querer mejorar como personas y profesionistas.

\section{MATERIAL Y MÉTODO}

Este trabajo fue desarrollado en la DACEA perteneciente a la UJAT. La investigación cualitativa utilizada en este trabajo, se maneja mediante la corriente epistemológica fenomenológica, la cual se preocupa por comprender los actores sociales y por ello de la realidad subjetiva (Martínez, 2011).

\section{Muestra}

El estudio empírico se basó en una muestra intencional o de conveniencia de 261 estudiantes de primero y segundo ciclo de las cuatro licenciaturas con que cuenta la División Académica de 


\section{UNIVERSITARIOS ACTORES EN LA PRÁCTICA DE VALORES}

un total de 1,250 alumnos, toda vez que se busca conocer la formación en valores con la que ingresan a la universidad, y la práctica que éstos le dan a los mismos. El criterio para determinarla, se basó en la elección de estudiantes quienes, al momento de administrar los cuestionarios, cursaran sus estudios en la modalidad presencial. Los cuestionarios fueron llenados por los propios estudiantes de forma anónima y voluntaria.

\section{Instrumento}

Se aplicó un cuestionario con opción de respuesta múltiple a 261 estudiantes, consta de seis preguntas que se enfocan a la frecuencia con que el universitario practica los valores.

\section{Procedimiento y análisis}

Se les hizo una serie de preguntas a los alumnos acerca de la frecuencia con que practican los valores hacia sus padres, profesores, compañeros, donde piensan que se forjan los valores, cual valor consideran de más importancia y si les han hablado de valores. Además, se utilizó una escala de medición (siempre, casi siempre, pocas veces y nunca).

\section{RESULTADOS}

A continuación, se muestran los resultados obtenidos del cuestionario que se aplicó a los 261 estudiantes del primero y segundo ciclo en la Ciudad del Conocimiento de la DACEA.

Siendo el $48 \%$ de los participantes hombres, mientras que el 52\% mujeres. Donde el $78 \%$ de los participantes es de primer ciclo y el $22 \%$ de segundo. Los estudiantes a quienes se les aplicaron los cuestionarios fueron de Administración, Contabilidad, Economía y Mercadotecnia, ocupando un porcentaje de participación de 32\% Administración, 27\% Contabilidad, 19\% Economía y $22 \%$ Mercadotecnia.

Domínguez (2014) considera que el desarrollo social propio de la juventud propicia el surgimiento de una concepción científica y moral donde los valores tienen gran importancia y que esta concepción contribuye al proceso de autodeterminación del joven en todos los ámbitos de su vida; por ello, la formación de valores cobra relevancia y por lo tanto es necesario enfatizar en los mismos. 


\section{UNIVERSITARIOS ACTORES EN LA PRÁCTICA DE VALORES}

Cuando se les preguntó con qué frecuencia practican los valores hacia sus padres, el 56\% de alumnos contestaron que siempre, $37 \%$ casi siempre, $6 \%$ pocas veces y el $1 \%$ que nunca.

Tabla 1

¿Con qué frecuencia practicas los valores hacia tus padres?

\begin{tabular}{lcc}
\hline & No. Alumnos & $\%$ \\
\hline Siempre & 147 & 56 \\
Casi siempre & 97 & 37 \\
Pocas veces & 15 & 6 \\
Nunca & 2 & 1 \\
Total & 261 & 100 \\
\hline
\end{tabular}

Fuente: Elaboración

propia.

También se les preguntó la frecuencia con que practican los valores hacia sus profesores, a lo cual el $56 \%$ de los alumnos señalaron siempre, 38\% casi siempre, $6 \%$ pocas veces y el $0 \%$ nunca.

Tabla 2

¿Hacia tus profesores?

\begin{tabular}{lcc}
\hline & No. Alumnos & $\%$ \\
\hline Siempre & 145 & 56 \\
Casi siempre & 100 & 38 \\
Pocas veces & 15 & 6 \\
Nunca & 1 & 0 \\
Total & 261 & 100 \\
\hline
\end{tabular}

Fuente: Elaboración propia.

A la pregunta, ¿hacia tus compañeros?, 22\% de los alumnos respondieron siempre, 49\% casi siempre, $25 \%$ pocas veces y $4 \%$ nunca. 
UNIVERSITARIOS ACTORES EN LA PRÁCTICA DE VALORES

Tabla 3

¿Hacia tus compañeros?

\begin{tabular}{lcc}
\hline & No. Alumnos & $\%$ \\
\hline Siempre & 58 & 22 \\
Casi siempre & 127 & 49 \\
Pocas veces & 65 & 25 \\
Nunca & 11 & 4 \\
Total & 261 & 100 \\
\hline
\end{tabular}

Fuente: Elaboración propia.

Sandoval (2014) señala que, para lograr una convivencia social, pacífica y democrática entre los miembros de la comunidad educativa, es necesario el aprendizaje y la práctica de valores. En ese sentido, la tarea de educar en valores no está circunscrita solo al ámbito escolar; la familia y la sociedad son espacios sociales fuertemente comprometidos en esta responsabilidad.

A la pregunta, sobre ¿dónde considera el universitario que se forjan los valores?, el 15\% de los alumnos optó por la escuela, 5\% por los amigos, 79\% por la familia y 1\% por la religión.

\section{Tabla 4}

¿Dónde consideras que se forjan los valores de los universitarios?

\begin{tabular}{lcc}
\hline & No. Alumnos & $\%$ \\
\hline Escuela & 39 & 15 \\
Amigos & 13 & 5 \\
Familia & 205 & 79 \\
Religión & 4 & 1 \\
Total & 261 & 100 \\
\hline
\end{tabular}

Fuente: Elaboración propia. 


\section{UNIVERSITARIOS ACTORES EN LA PRÁCTICA DE VALORES}

La valoración es un proceso subjetivo que refleja la significación que poseen estos objetos para el sujeto y como proceso subjetivo es acertada cuando se corresponde con el valor; es decir, cuando refleja la significación socialmente positiva de algo (Avelino, Fierro y Acosta, 2014).

En la pregunta que se les hizo a los universitarios para saber ¿qué valor es de más importancia para ellos?, el $46 \%$ respondió que el respeto, $27 \%$ la honestidad, $10 \%$ la igualdad y $17 \%$ la responsabilidad.

Tabla 5

De los siguientes valores señala cuál es el más importante para ti

\begin{tabular}{lcc}
\hline & No. Alumnos & $\%$ \\
\hline Respeto & 119 & 46 \\
Honestidad & 72 & 27 \\
Igualdad & 26 & 10 \\
Responsabilidad & 44 & 17 \\
Total & 261 & 100 \\
\hline
\end{tabular}

Fuente: Elaboración propia.

El personal docente universitario en México transita como un mediador entre los simbolismos, percepciones, costumbres y progresos, pasados y presentes en el colectivo social, y el desarrollo extraordinario y vertiginoso que se sucede e incide en las nuevas generaciones (Cantú, 2018).

Los alumnos respondieron que la frecuencia con la que los profesores les hablan de valores es, $24 \%$ siempre, $39 \%$ casi siempre, $32 \%$ pocas veces y $5 \%$ nunca. 


\section{UNIVERSITARIOS ACTORES EN LA PRÁCTICA DE VALORES}

Tabla 6

¿Con qué frecuencia tus profesores te han hablado de los valores?

\begin{tabular}{lcc}
\hline & No. Alumnos & $\%$ \\
\hline Siempre & 63 & 24 \\
Casi siempre & 101 & 39 \\
Pocas veces & 84 & 32 \\
Nunca & 13 & 5 \\
Total & 261 & 100 \\
\hline
\end{tabular}

Fuente: Elaboración propia.

\section{DISCUSIÓN}

Los valores son esenciales en la formación de los universitarios, y en ella interviene la familia, la escuela, la religión y los amigos, pero por alguna razón se puede observar en la tabla 1, que el 44\% de los alumnos no siempre practican los valores hacia sus padres, ya que sólo el 56\% de los universitarios señalaron que siempre lo hacen, y tomando en cuenta que los valores son producto de las experiencias, podemos decir que el crecimiento de los universitarios ha carecido de una enseñanza en valores.

Cuando respondieron a la práctica de valores hacia sus profesores, el 0\% de los universitarios señalaron que nunca lo hacen; por lo tanto, aunque no el $100 \%$ de ellos lo hacen siempre, es notable que así sea en mediadas diferentes, todos los universitarios practican los valores.

En la tabla 4, el 79\% de los universitarios señalaron que los valores se forjan en la familia. Palacios y Oliva (2003) en Guzmán y Pacheco (2014) mencionan que la actitud que los padres transmiten a sus hijos hacia la educación, la cultura, los profesores y la escuela (institución) ejercen gran influencia en el proceso de aprendizaje.

El análisis de los valores, se realiza a través de combinaciones particulares seleccionadas por cada persona, que muestran su forma de entender el mundo y de actuar (Elexpuru, Villardón y Álvarez, 2013). Así, en la tabla 5 sobresale el valor respeto como el más importante para los universitarios. 


\section{UNIVERSITARIOS ACTORES EN LA PRÁCTICA DE VALORES}

La comunicación entre profesores y alumnos debe ser más fuerte, para que de esta forma se facilite entablar pláticas en relación no sólo a los valores sino a cualquier tema de importancia, que pueda contribuir en el crecimiento de los estudiantes. Asimismo, la tabla 6 refleja la baja frecuencia con que los profesores hablan de valores con los alumnos.

Durante el aprendizaje es prioritario el papel de la universidad, como lugar en el que se adquieren y modifican valores y actitudes a través de sus experiencias en interacción con sus profesores y compañeros (Morales, Infante y Trianes, 2013).

\section{CONCLUSIONES}

Con base en los resultados obtenidos, se puede afirmar que los universitarios necesitan ser conscientes sobre la importancia que tienen los valores, tanto para su profesión como para su vida personal, ya que se aprecia la falta de práctica de valores, siendo ésta hacia los padres, profesores o compañeros.

Hablar de valores parece ser visto como un tabú en la sociedad, y por ello, no se le da la debida importancia, lo que nos vuelve incapaces de ver el impacto que tiene en el actuar de cada persona.

La aplicación de los cuestionarios brindó información que permitió alcanzar el objetivo del trabajo de investigación, debido a que se logró exponer la formación en valores que los recién ingresados a la universidad traen de las instituciones educativas anteriores, mediante el reflejo de la práctica de valores que realizan hacia las personas con las que conviven actualmente.

Los universitarios coinciden en que los valores se forjan en el hogar; es decir, dentro del núcleo familiar, y como se expuso, varios autores coinciden en ello, pero hay que reconocer que en la formación de los valores no solamente es responsable la familia sino también las entidades educativas, la religión y los amigos, todos aquellos ámbitos en los que el joven universitario se interrelaciona en el día a día, y que lo moldea para ser una persona con criterio propio.

El respeto y la honestidad son los valores que los universitarios señalaron como más importantes, estos son dos de los valores que ayudan a preservar una convivencia armónica y pacífica, y que encamina a la práctica de los demás valores. 


\section{UNIVERSITARIOS ACTORES EN LA PRÁCTICA DE VALORES}

\section{REFERENCIAS BIBLIOGRÁFICAS}

Aranda, J. y Salgado, E. (2005). La formación de valores en el ser humano. Innovación Educativa, 5(28), pp. 33-43. Recuperado de http://www.redalyc.org/articulo.oa?id=179421470004.

Avelino, C., Fierro, B. y Acosta, H. (2014). El desarrollo de los valores en los estudiantes de Ciencias de la Educación de la Universidad José Eduardo Dos Santos, Angola. Atenas, 1(25), pp. 127-139. Recuperado de http://www.redalyc.org/pdf/4780/478047201008.pdf.

Bolívar, A. (2005). El lugar de la ética profesional en la formación universitaria. Revista Mexicana de Investigación Educativa, 10(24), pp. 93-123. Recuperado de http://beu.extension.unicen.edu.ar/xmlui/handle/123456789/273.

Cantú, P. (2018). Profesorado universitario: Emisor de valores éticos y morales en México. Revista Educación, 42(1). Recuperado de http://www.scielo.sa.cr/pdf/edu/v42n1/22152644-edu-42-01-00105.pdf.

De los Ríos, A., Millán, S., Ruiz, M. y Tirado, P. (2015). Los valores en el título de administración y dirección de empresas: un análisis de su importancia para estudiantes y egresados. Revista de Investigación Educativa, 33(2), pp. 385-399. Recuperado de http://www.redalyc.org/pdf/2833/283341409009.pdf.

Domínguez, L. (2014). La formación de valores en jóvenes universitarios. Universidad de La Habana, (278), pp. 108-118. Recuperado de http://scielo.sld.cu/pdf/uh/n278/uh07278.pdf.

Elexpuru, I., Villardón, L. y Álvarez, C. (2013). Identificación y desarrollo de valores en estudiantes universitarios. Revista de Educación, (362). Recuperado de http://www.revistaeducacion.educacion.es/doi/362_158.pdf.

González, V. (2000). Enfoque psicopedagógico para su estudio. Revista cubana educación médica superior, 14(1), pp. 74-82. Recuperado de http://scielo.sld.cu/pdf/ems/v14n1/ems10100.pdf.

González, R. y Cardentey, J. (2016). Educación en valores de estudiantes universitarios. Humanidades médicas, 16(1), pp. 161-174. Recuperado de http://www.medigraphic.com/pdfs/hummed/hm-2016/hm161k.pdf. 


\section{UNIVERSITARIOS ACTORES EN LA PRÁCTICA DE VALORES}

Guzmán, R. y Pacheco, M. (2014). Comunicación familiar y desempeño académico en estudiantes universitarios. Redalyc.org, (20), pp. 79-91. Recuperado de http://plataforma.responsable.net/sites/default/files/comunicacion_familiar_y_desempen o_academico_3.pdf.

Morales, F., Infante, L. y Trianes, M. (2013). Perfiles de valores éticos en estudiantes universitarios. Aula abierta, 41(2), pp. 55-66. Recuperado de file:///D:/DESCARGAS/Dialnet-PerfilesDeValoresEticosEnEstudiantesUniversitarios4239108.pdf.

Martínez, M. (2006). Formación para la ciudadanía y educación superior. Revista Iberoamericana de Educación, (42), pp. 85-102. Recuperado de https://upcommons.upc.edu/bitstream/handle/2099/9316/article\%20Miquel\%20Martinez .pdf.

Mazadiego, T. (2005). Propuesta humanista para la clarificación de valores en estudiantes universitarios (tesis para obtener el grado de doctor en investigación psicológica). Universidad Iberoamericana Departamento de Psicología. México.

Mejía, C. (2007). Formación humanística universitaria: reto y urgencia de los tiempos modernos. $\begin{array}{lllll}\text { Sophia, } \quad \text { (3), } & \text { 81-87. } & \text { Recuperado }\end{array}$ http://www.redalyc.org/pdf/4137/413740746007.pdf.

Montesinos, E. (2010). Los valores en la formación de los universitarios desde la construcción de su identidad. Recuperado de https://www.gestiopolis.com/los-valores-en-laformacion-de-los-universitarios-desde-la-construccion-de-su indentidad/.

Riera, L. y Sansevero, I. (2013). El compromiso ético del estudiante universitario de las experiencias de aprendizaje-servicio. Omnia, 19(3), pp. 31-42. Recuperado de http://www.redalyc.org/pdf/737/73730059004.pdf.

Sandoval, M. (2014). Convivencia y clima escolar: claves de la gestión del conocimiento. Última década, (41), pp. 153-178. Recuperado de file:///D:/DESCARGAS/DialnetConvivenciaYClimaEscolar-5287732.pdf. 